\title{
A Study on the Vertical Navigation of High Rise Buildings
}

\author{
Xianghong Sun ${ }^{1}$, Tom Plocher ${ }^{2}$, and Weina $\mathrm{Qu}^{1}$ \\ ${ }^{1}$ State Key Laboratory of Brain and Cognitive Science, Inst. of Psychology, \\ Chinese Academy of Science, \\ Beijing 100101, China \\ ${ }^{2}$ Honeywell ACS Labs, \\ Minneapolis MN 55418, USA \\ tom.plocher@honeywell.com
}

\begin{abstract}
Scroll bar and stab touch screen controls were designed and compared to a soft keyboard to help firefighters perform vertical navigation tasks for high-rise buildings depicted on a graphical display. 18 male subjects were asked to accomplish three experimental tasks: 1) input floor number and navigate to the floor, 2) switch the current floor to another one that was two floors higher/lower, and 3) check around the floors in the high rise to find the one on fire. Task completion time and number of screen operations were recorded. Results showed that, keyboard method of floor selection was the fastest, and scroll bar the slowest. In Task 1 and Task 2, the least number of screen operations were shown with soft keyboard. But in Task 3, keyboard was slowest. The other two control methods were not sensitive to tasks. Design implications for scroll bar and stab controls are discussed.
\end{abstract}

Keywords: touch screen, vertical navigation, scroll bar, graphical displays.

\section{Introduction}

In order to detect the original fire and the current fire spread in a high rise building as quickly and accurately as possible, intelligent fire information display systems should show firefighters detailed information floor by floor, such as the floorplan, the location of a fire, the current position of firefighters, and the locations of fire hydrants, water pipes, stairs, and dangerous materials. An example of such a display is shown in Figure 1. The fire information display must allow firefighters to switch between floors very easily to view the floor plan and associated information objects. What is an intuitive way for firefighters to input the desired floor number or navigate vertically through the floors of the building searching for the source of the fire? The situation is analogous to that of people using an elevator. But, unlike an elevator control panel, a computer touch screen does not have unlimited space for buttons. For buildings higher than ten floors, there simply is not enough screen space to assign a single button to each individual floor. 
Vertical navigation techniques that are effective, but much more efficient in their use of screen space are needed[1,5,6]. In this study, we designed several kinds of vertical navigation controls for a touch screen display, and determined which one was the best for fire fighters to use for gathering information from the graphical floor plans.

From existing computer interface designs, we have found several possible ways to support the fire fighter's task of switching between floors and floor plans[2,3].

First, the scroll bar has been widely used for moving between different parts of a document. For our vertical scroll bar control, we assumed that the location of the slide represents the floor level. The higher the slide position is on the scroll bar, the higher the floor level. It bears a quite strong analogy to a real high-rise building that we assumed should be reflected in ease of use.

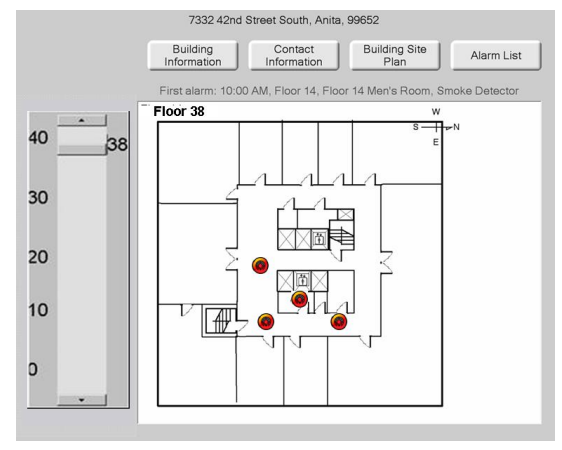

Fig. 1. Typical firefighter information display (with experimental scrollbar control)

A second approach is the stab control. Typically, the stab control is used in a submenu to list all the functions when the functions and operations are too numerous to display in one page and when some of them can be grouped together. Typically, the stab control is presented in a horizontal way. For our application it was positioned vertically. One stab was assigned to each floor level. The selected floor plan was shown in full size, and the others half hidden and overlapped behind the selected floor plan. The user switches floor levels by clicking the corresponding stab on the screen.

Keyboards, our third approach, are used to accurately input or set a value, such as setting the font size at 11.5 points. With mobile phones, access control systems, etc., it is easy to find keyboards in daily life. Even people who lack computer experience know how to use a keyboard to input digits. If a fire fighter wanted to go to a specific floor, such as the 14th floor, he or she would simply type in "14", then press "enter". But one problem with keyboards is that it is difficult to change the current number to another one even if there is only one digit difference between the two numbers. One would question how suitable it is for a task that frequently involves rapidly changing the number up and down one digit at a time. We would predict that it is not very well suited to the fire fighter display task of switching floors.

Based on interviews with Chinese firefighters, it was found that they have three main tasks that require vertical navigation through the building floor plans: 1) to check floor by floor and find which floors are involved in the fire, 2) to switch between floors 
and find the stairways, and 3) to go to a specific floor for a detailed description of key objects. The three vertical navigation designs are shown in Figure 2. The left one is the scroll bar that includes a movable slide and two up/down arrow buttons. The slide could be dragged to any specific floor level and the current floor number shown beside the slide. The up/down arrow buttons could make the slide one floor higher or lower. The middle one is the stab display that looks like a set of cards shown vertically on screen. The current floor and two adjoined floors are shown on cards. The other cards were overlaid behind the current floor card. There was also a pair of up/down arrow buttons at the two ends of the stab to make the current floor card go up/down by one floor. To the right is shown the soft keyboard display that included 10 digit buttons and two left/right arrow buttons. People can press the digits on the screen to get to a specific floor, and press the left/right arrow to go up/down by one floor.

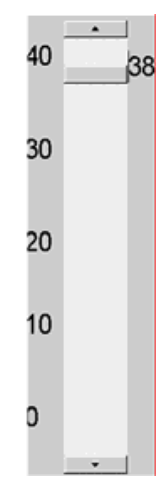

(a) scroll bar

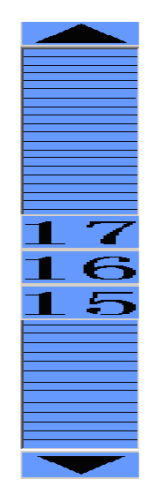

(b) stab control

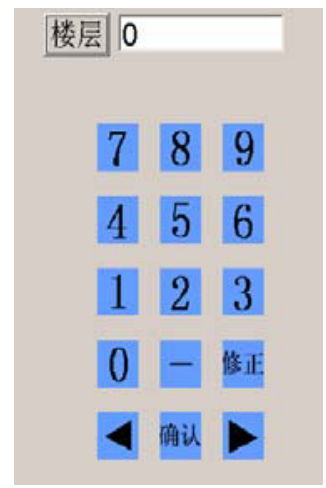

(c) soft keyboard on screen

Fig. 2. Three vertical navigation control designs

Comparing the three kinds of vertical navigation methods, the following questions need to be answered. 1) Which method is the fastest for helping firefighters select a specific floor? 2) Which method is the fastest for switching between floors? 3) Which method is the easiest for moving up/down by just one floor? We hypothesized that since the scroll bar is easy to learn and has a strong analogy to a high-rise building, it would be the best method for the vertical navigation.

\section{Method}

\subsection{Experimental Design}

The independent variable consisted of three kinds of vertical navigation methods: keyboard, vertical scroll bar, and stab control. The dependent variables were task completion time and the time to complete screen operations for each task, including clicking a button, and dragging a finger on screen. 


\subsection{Experimental Tasks}

Corresponding to the three questions mentioned above, there were three experimental tasks:

Task 1. Move to a specific floor level by inputting the floor number as directed.

Task 2. Switch to another floor level that is two floors higher or lower than current position. This task was designed for firefighters to check detailed information at adjacent floors. Different from the Task 1, this task displayed the current floor number on the screen, and asked subjects to change the current floor to another one. For the soft keyboard display, the subjects had to delete the current number, and then input another one. Another way to use the keyboard for this was to click the left/right arrow button to make the floor number increase/decrease by one floor. For the scroll bar, subjects needed to drag the slide from the current position/floor to the requested floor level, or press the up/down button to make the slide move two floors. The stab control was operated in a manner similar to the scroll bar control.

Task 3. Check floors to find the fire. Subjects were told that the fire went off between Floor A and Floor B, and were asked to find it. For all three vertical navigation methods, one way to find the fire was to press the up/down button to check each floor until the fire was found. For the scroll bar and stab control an additional method was to drag the slide or current floor card smoothly, until the fire was found. For the soft keyboard display an additional method was to delete the current floor number and input a new one to find the fire.

\section{Procedure}

Without any training, subjects were asked to accomplish the three experimental tasks one by one. The order of the three tasks was counter-balanced by Latin square between subjects. For each task, subjects were asked to accomplish by the following three ways respectively and randomly: keyboard, scroll bar, and stab control. Each part included 20 trials. After a total of 60 trials, a short subjective evaluation about the three kinds of vertical displays was conducted. During the test, screen resolution was set at 1280 by 1024 .

Participants: Since all the firefighters in China are male soldiers, and their age is around 18 to 36 years, 14 male subjects at the same age participated in this experiment. Each subject took half an hour to accomplish the three experimental tasks. This was followed by a 5-minute interview.

\section{Results}

\subsection{Practice Effect}

The interface of the Fire Information Display System should not require any training. So in this study, subjects didn't get any training, or introduction before the test. They had to guess how to accomplish the tasks. Subjects had 20 trials of each task. The task completion time and the number of control operations were recorded as their performance. Since there were some data lost at the 10th and the 20th trial, only the first 9 performances were analysed. 


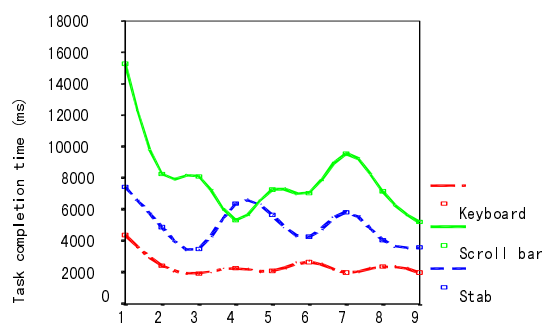

(a) Task 1. Move to a specific floor

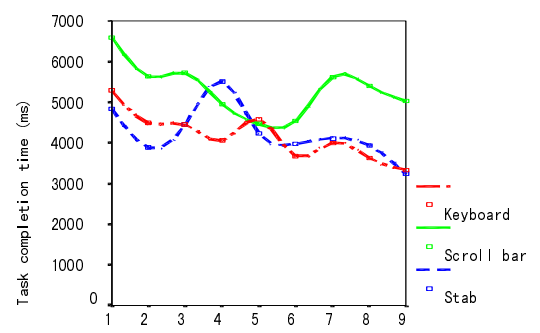

(b) Task 2. Switch to another floor

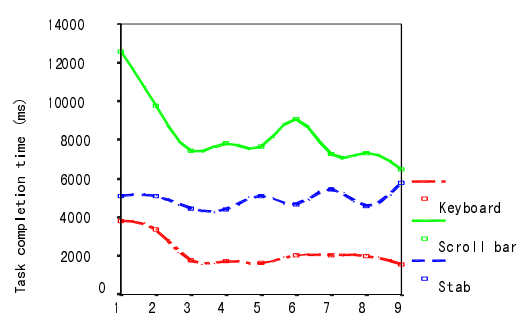

(c) Task 3. Check floors to find the fire

Fig. 3. Practice effects on the three experimental tasks

Fig. 3 (a)- (c) shows the different practice patterns when people use soft keyboard, scroll bar, or stab control as the vertical navigation method to accomplish the three experimental tasks. For Task 1 (finding a specific floor as directed; see Fig. 3 (a)), the first trial took significantly longer than the other 8 trials $(F(2,8)=4.196, p<.001)$. The task completion time (TCT) using the soft keyboard kept stable around 2 seconds, and the TCT for the scroll bar and stab control fluctuated between 8 seconds and 4 seconds. However, these differences across the eight trials were not significant. In other words, people needed only one trial to learn how perform Task 1 with all three kinds of floor selection methods.

For Task 2 (switching from current floor to two floors higher/lower; see Fig. 3 (b)), there was no difference across the 9 trials. This means that after the Task 1 was completed, Task 2 didn't need any further training to use soft keyboard, scroll bar and stab. But the TCT of all three vertical navigation methods fluctuated between 6 and 4 seconds. 
For Task 3 (check floors and find the fire; see Fig. 3 (c)), the TCT of using soft keyboard still was stable at 2 seconds, TCT of stab at 5 seconds. But on the first two trials, the TCT of using the scroll bar was significantly longer than the TCT of the other 7 trials. The amount of training required for the scroll bar in Task 3 was two times that for the other methods.

\subsection{Task Completion Time}

Since after the first two trials, there was no significant difference among the other trials, the mean of TCTs in the last 16 trials was calculated as each subject's performance. The

Table 1. Mean task completion times (msec.) for three vertical navigation controls

\begin{tabular}{lllll}
\hline \multirow{2}{*}{ Task } & \multicolumn{2}{l}{ Vertical navigation } & & \\
\cline { 2 - 4 } & Soft keyboard & Scroll bar & Stab & $\mathrm{p}$ \\
\hline 1 & 2181.9 & 7440.8 & 4907.9 & $\mathrm{~F}(2)=93, \mathrm{p}<.01$ \\
2 & 3362.4 & 4760.6 & 4096.4 & $\mathrm{~F}(2)=6.4, \mathrm{p}<.01$ \\
3 & 1772.7 & 8284.5 & 4925.1 & $\mathrm{~F}(2)=83.7, \mathrm{p}<.01$ \\
$\mathrm{p}$ & $\mathrm{F}(2)=11.8, \mathrm{p}<.00$ & $\mathrm{~F}(2)=23, \mathrm{p}<.01$ & & \\
\hline
\end{tabular}

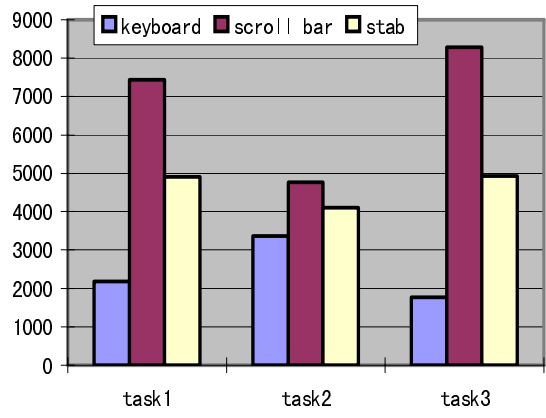

(a) Task completion time

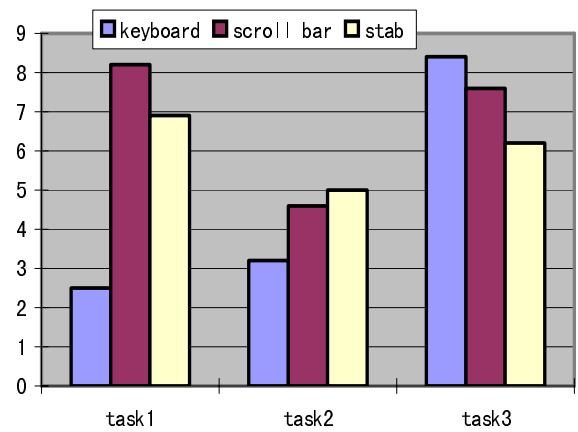

(b) numbers of screen operations during each trial

Fig. 4. Comparison of three vertical navigation controls on different tasks 
average TCT under different experimental situations are listed in Table 1. All the TCT ranged from $1700 \mathrm{~ms}$ to $8300 \mathrm{~ms}$. Based on the ANOVA for repeated measures, the scroll bar display made the TCT the longest in all three tasks, and the soft keyboard made it the shortest ( $F$ values and $p$ values are shown in Table 1). The TCT under stab situation kept stable at $4000 \mathrm{~ms}$ to $5000 \mathrm{~ms}$ (also see Fig.4 (a)). This result was just opposite to the hypothesis we made, which probably because that people are much more familiar with keyboard than with scroll bar and stab. Scroll bar and stab still were quite new things for subjects. Especially people didn't use them to input information to computer system. In addition, when using scroll bar to locate a position, people usually took two steps: first to drag the slide to a position roughly, then move the slide very slow and adjust the position very carefully, or use up/down button to adjust the position with very small steps. But in this study, it's found that some subjects didn't use up/down button, it took subjects long time to drag the slide and adjust the position.

\subsection{Number of Screen Operation}

Numbers of screen operations were recorded as another criterion on which to compare the three vertical navigation methods. Sometimes people took a shorter time to input a floor number, or switch floors, but the way of vertical navigation made people touching the screen button or moving their finger on the screen many times to accomplish a simple task. It didn't mean easy and intuitive.

Table 2 lists the average number of screen operations under different experimental conditions. The soft keyboard cost the least in operation time on Task 1 and Task 2 . There was no difference in number of operations between the stab control and scroll bar. The keyboard was always the best way to input information accurately, and the scroll bar and stab control needed more individual input operations to adjust their positions very carefully. But for the Task 3, which involved checking floors to find the fire, the soft keyboard lost its advantage. The number of operations for the keyboard method increased dramatically, owing to the requirement to change floors very frequently. In addition, some subjects didn't notice the left and right arrow buttons that could have helped them change floors very quickly. The scroll bar and stab control were not sensitive to different tasks including Task 3.

Table 2. Numbers of screen operation

\begin{tabular}{ccccc}
\hline & \multicolumn{3}{c}{ Vertical navigation } & \\
\cline { 2 - 4 } Task & Soft keyboard & Scroll bar & Stab & $\mathrm{p}$ \\
\hline 1 & 2.5 & 8.2 & 6.9 & $\begin{array}{c}\mathrm{F}(2)=4.65, \\
\mathrm{p}=.014\end{array}$ \\
2 & 3.2 & 4.6 & 5.0 & $\begin{array}{c}\mathrm{F}(2)=4.09, \\
\mathrm{p}=.025\end{array}$ \\
3 & 8.4 & 7.6 & 6.2 & No difference \\
$\mathrm{p}$ & $\begin{array}{c}\mathrm{F}(2)=38 ., \\
\mathrm{p}<.00\end{array}$ & No difference & $\begin{array}{c}\text { No } \\
\text { difference }\end{array}$ \\
\hline
\end{tabular}




\subsection{Subjective Evaluation}

After the test, 16 subjects accepted a 5-minute follow-up. They were asked to answer the following questions:

1) For Task 1, which way do you think is the best way to input the floor number and get to the floor? Which one is the worst? Why?

2) For Task 2, and Task 3, which way do you think is the best way to check floors and find the fire? Which one is the worst? Why?

3) Did you use the up/down arrows during the test?

4) Are there some features you think could be improved?

For questions 1 and 2, the most preferred navigation method received a score of 3 and the least preferred received a score of 1 . The average scores for the three methods of vertical navigation are shown in Table 3. It showed that people did not prefer any one of the vertical navigation methods to another on tasks 2 and 3. But they did prefer using the soft keyboard to input a floor number in Task 1.

In addition, five subjects said they didn't notice there were up/down arrow buttons at the two ends of scroll bar and stab control. Another six subjects didn't notice that there were left/right arrow buttons on the soft keyboard interface. Up/down buttons were critical for people to adjust the current position by one floor. The result showed that the up/down buttons were not apparent enough to draw people's attention. Some subjects suggested making the size of up/down buttons a little bigger than they were, or make their colour different from other buttons.

Most people liked the scroll bar display, but felt that the current implementation was not good enough. For example, the slide was not sensitive enough to the finger's dragging movements. Subjects always complained that the slide didn't follow their finger going down/up quickly and smoothly. It made them feel frustrated. It probably was the main reason why the scroll bar display didn't show more advantages than the soft keyboard. This suggests that we improve the current design and conduct another experiment in the future.

People liked the stab control too, but they felt the interval between two floors was too narrow for their fingers to distinguish.

Table 3. Subjective evaluations of three kinds of vertical navigation controls

\begin{tabular}{ccccc}
\hline & \multicolumn{3}{c}{ Vertical navigation } & \\
\cline { 2 - 5 } Task & Soft keyboard & Scroll bar & Stab & $\mathrm{p}$ \\
\hline 1 & 2.5 & 1.7 & 1.8 & $\mathrm{~F}(2)=4.8$, \\
$\mathrm{p}=.026$ \\
N,3
\end{tabular}

\section{Discussion and Conclusion}

In this experiment, the soft keyboard was superior to the scroll bar and stab controls for vertical navigation of a high rise building on a touch screen. We suspect that there were two reasons for this:

1. With no training on the three navigation controls prior to performing the high rise navigation tasks, the keyboard simply was the most familiar way for 
subjects to input information. Everyone, including people who lack of computer experience, and know how to use a keyboard. It has been standardized and is widely accepted.

2. The scroll bar design used in the study needs to be improved. The implementation used in the experiment was not sensitive enough for a touch screen. In particular, the slide in the scroll bar proved to be not big enough. A slide bigger than 50 X 20 pixels needs to be designed and tested. The Stab control suffered from similar problems.

Although the scroll bar and stab controls produced lesser performance than the keyboard for high rise vertical navigation, the subject's comments indicated that, with some re-design, these might be valid alternatives to the keyboard for touch screens. It should be noted that the scroll bar and stab control could be used as displays also. That is, they could provide an intuitive way to indicate the currently selected floor position in a high-rise building. Further exploration of this is left for the future study.

Acknowledgments. Thanks to the people from Beijing Fire-Fighting Bureau who gave us access and helped us in this study.

\section{References}

1. Aftab, E.P, Sebastian, S., Tomescu, G.A., M.: Ishac: What visual information is used for navigation around obstacles in a cluttered environment? Canada Journal of Physiology and Pharmacology 82, 682-692 (2004)

2. Vries, d., Bauke, Buma, Sjoerd, Jessurun, Joran.: An Intuitive Interface for Building Management and Planning. International Journal of Architectural Computing 4(2), 17-26 (2006)

3. Feary, M., Alkin, M., Polson, P., McCrobie, D., Sherry, L., Palmer, E.: Aiding vertical guidance understanding. Air. \& Space Europe 1(1), 38-41 (1999)

4. Fraser, Peter, J.: Review: Depth, navigation and orientation in crabs: Angular acceleration, gravity and hydrostatic pressure sensing during path integration. Marine and Freshwater Behaviour and Physiology 39(2), 87-97 (2006)

5. Yao, G.: Application of touch screen in HCI. Construction Machinery \& Maintenance 10, 16-20 (2004)

6. Zhang, Y., Zhang, Z., Zhang, J., Wu, J.: 3D Building Modelling with Digital Map, Lidar Data and Video Image Sequences. The. Photogrammetric Record 20(111), 285-302 (2005) 